\title{
One-Year Clinical Evaluation of the Bonding Effectiveness of a One-Step, Self-Etch Adhesive in Noncarious Cervical Lesion Therapy
}

\author{
Babacar Faye, Mouhamed Sarr, Khaly Bane, Adjaratou Wakha Aidara, \\ Seydina Ousmane Niang, and Abdoul Wakhabe Kane \\ Department of Conservative Dentistry and Endodontics, University of Cheikh Anta Diop-Dakar, BP 5005, Dakar, Senegal \\ Correspondence should be addressed to Babacar Faye; babsfaye@yahoo.com
}

Received 10 November 2014; Revised 10 February 2015; Accepted 13 February 2015

Academic Editor: Ali I. Abdalla

Copyright (C) 2015 Babacar Faye et al. This is an open access article distributed under the Creative Commons Attribution License, which permits unrestricted use, distribution, and reproduction in any medium, provided the original work is properly cited.

\begin{abstract}
This study evaluated the one-year clinical performance of a one-step, self-etch adhesive (Optibond All-in-One, Kerr, CA, USA) combined with a composite (Herculite XRV Ultra, Kerr Hawe, CA, USA) to restore NCCLs with or without prior acid etching. Restorations performed by the same practitioner were evaluated at baseline and after 3, 6, and 12 months using modified USPHS criteria. At 6 months, the recall rate was 100\%. The retention rate was $84.2 \%$ for restorations with prior acid etching, but statistically significant differences were observed between baseline and 6 months. Without acid etching, the retention rate was $77 \%$, and no statistically significant difference was noted between 3 and 6 months. Marginal integrity ( $93.7 \%$ with and $87.7 \%$ without acid etching) and discoloration (95.3\% with and $92.9 \%$ without acid etching) were scored as Alpha or Bravo, with better results after acid etching. After one year, the recall rate was 58.06\%. Loss of pulp vitality, postoperative sensitivity, or secondary caries were not observed. After one year retention rate was of $90.6 \%$ and $76.9 \%$ with and without acid conditioning. Optibond All-in-One performs at a satisfactory clinical performance level for restoration of NCCLs after 12 months especially after acid etching.
\end{abstract}

\section{Introduction}

Currently available adhesives can be categorized into two groups according to the adhesive and clinical application: (i) the etch-and-rinse group, in which a separate etchant is applied and rinsed off, and (ii) the self-etch group, in which an acidic monomer is used to simultaneously demineralize and infiltrate the tooth surface. These adhesives have been simplified and improved to provide better long-term performance [1]. Self-etch adhesives were introduced as an alternative to etch-and-rinse adhesives because of their reduced technique sensitivity and application time $[1,2]$. The in vitro evaluation of self-etch adhesives by many researchers has led to high values of microtensile bond strength $(\mu \mathrm{TBS})$, particularly when they are applied to dentin [3-6].

However, a direct correlation between laboratory and clinical results has not yet been shown. Thus, it is difficult to transfer these laboratory results to the clinical setting, where there are many confounding factors. Peumans et al. used class $\mathrm{V}$ restorations to clinically evaluate adhesive systems [1]. Noncarious cervical lesions (NCCLs) (including erosion, abrasion, and abfraction) are types of chronic tooth surface destruction that are not bacterial in origin $[7,8]$. With increases in the aged population and therefore the number of people who retain their teeth for long periods of time, the prevalence of NCCLs is increasing [8]. The procedures used to treat NCCLs provide excellent opportunities for the evaluation of new materials, because little lesion preparation is needed, and access and restoration are simple. Additionally, the restorations are not load-bearing and thus provide an ideal surface to evaluate the potential of a new adhesive [7]. Furthermore, many clinical studies in conservative dentistry have focused on the treatment of NCCLs because of their increased prevalence and aesthetic implications. Previous 


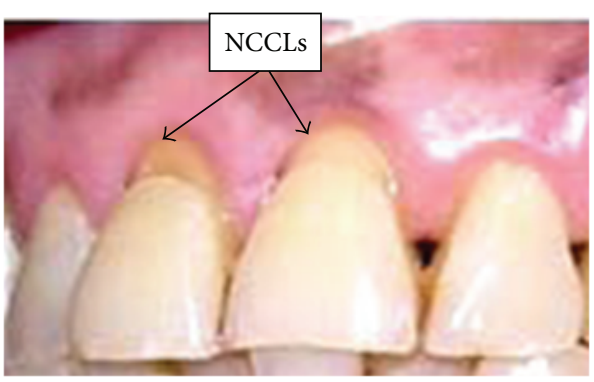

(a) Before treatment

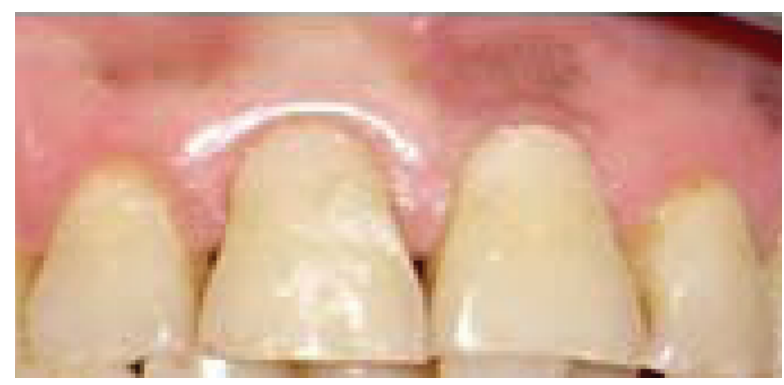

(b) After treatment (baseline)

FIgURE 1: A front view of NCCLs in 11 and 21 before and after restoration.

results indicated that two-step, self-etch adhesive performs reliably and stably in the clinic [1].

The aim of this study was to evaluate the one-year clinical performance of a one-step, self-etch adhesive system (Optibond All-in-One) combined with Herculite XRV Ultra composite with or without prior etching of the enamel margins in the restoration of NCCLs, using modified United States Public Health Service (USPHS) criteria.

\section{Materials and Methods}

Thirty-one volunteer patients from the operative dentistry clinic at Dakar University (Senegal) were included in this study. The volunteers included 26 males and 5 females who ranged in age from 24 to 73 years. These patients were properly informed about the study, and those who agreed to participate signed a consent form that had been reviewed and approved by the Senegalese National Committee of Ethics. The criteria for inclusion in this study were appropriate oral hygiene, low level of dental decay, absence of periodontal disease, absence of bruxism, and the presence of at least two NCCLs to be restored. All subjects presented a minimum of one pair of NCCLs with a depth greater than or equal to $1 \mathrm{~mm}$.

The NCCLs were restored by the same dental practitioner using self-etch adhesive (Optibond All-in-One, Kerr, Orange, CA, USA) combined with restorative composite (Herculite XRV Ultra, shade A2, Kerr Hawe, Orange, CA, USA). No cavity preparation was performed, and a rubber dam was employed in all cases to prevent contamination.

All patients received symmetrical restorations. On one side, the enamel was etched for 30 seconds with a $36 \%$ phosphoric acid gel (De Trey Conditioner 36, Dentsply De Trey, PA, USA). The adhesive was applied in strict accordance with the manufacturer's instructions and light-cured for 20 seconds. The resin composite (Herculite XRV Ultra) was applied using a minimum of two increments and each increment was light-cured for 40 seconds. Any excess composite was removed with a diamond bur, and the restoration was finished with polishing discs (3M Espe, St. Paul, MN, USA). On the opposite side, the same steps were followed without prior etching of the enamel margins.

The practitioner was not involved in the evaluation of the restorations. At 3, 6, and 12 months following restoration, the clinical effectiveness was determined by two qualified evaluators using modified USPHS criteria (Table 1). The evaluators were blinded to the adhesive technique used in any given restoration. Any discrepancy between evaluators was resolved at chair side. Clinical effectiveness was determined in terms of the abovementioned parameters, of which retention (no complete loss of restoration), marginal integrity (severe defects), and clinical microleakage (severe discoloration) were considered as key parameters, determining the overall parameter "clinical success rate."

The chi-square test was used in the statistical analysis, and $P<0.05$ was considered to be statistically significant. Statistical analyses were performed using EPI INFO version 6, SPSS 16.0, and Microsoft Excel 2003 software.

\section{Results}

At baseline, all restorations were $100 \%$ successful with regard to the criteria evaluated (retention, marginal integrity, marginal discoloration, and tooth vitality) (Figure 1).

When comparing the criteria at 3 and 6 months, the retention was significantly different only for the restorations performed with prior acid etching of enamel margins $\left(\chi^{2}=\right.$ 4.47; $P=0.0344)$ (Table 2).

At 12 months, 18 out of 31 patients returned for examination, and the recall rate was therefore $58.06 \%$. Out of these patients, 82 teeth were examined, and $100 \%$ tooth vitality was recorded for all restorations with or without prior acid etching (Table 2).

After one year retention rate was respectively of $90.6 \%$ and $76.9 \%$ with and without acid conditioning (Table 3 ). The performance of restorations at the baseline and after 3,6 , and 12 months of recall time was evaluated by Mc Nemar's test $(\alpha=0.05)$ (Table 4$)$.

\section{Discussion}

The clinical effectiveness of various types of available resinbased adhesives has been reviewed previously [9]. It was concluded that the three-step etch-and-rinse systems provided the most reliable results, and the two-step self-etching systems had good potential $[10,11]$. There is a paucity of clinical trial data for All-in-One systems, preventing researchers from making definitive recommendations on these systems [1214].

In the current study, we evaluated the clinical performance of a one-step, self-etch adhesive combined with a 
TABLE 1: Modified USPHS rating criteria (de Munck et al.) [5].

\begin{tabular}{lcc}
\hline Category & Rating & Criteria \\
\hline \multirow{3}{*}{ Retention } & Alpha (A) & Retained \\
& Bravo (B) & Partially retained \\
& Missing & Charlie (C) \\
Marginal integrity & Alpha (A) & Closely adapted, no visible crevice \\
& Bravo (B) & Visible crevice, explorer will penetrate \\
& Charlie (C) & Crevice in which dentin is exposed \\
\hline \multirow{3}{*}{ Marginal discoloration } & Alpha (A) & No discoration \\
& Bravo (B) & Superficial staining (without axial penetration) \\
Tooth vitality & Charlie (C) & Deep staining (with axial penetration) \\
\hline \multirow{2}{*}{ Postoperative sensitivity } & Alpha (A) & Present \\
& Charlie (C) & None \\
\hline \multirow{2}{*}{ Secondary caries } & Alpha (A) & None \\
& Charlie (C) & Present \\
\hline
\end{tabular}

TABLE 2: Three- and six-month evaluations of restorations.

\begin{tabular}{|c|c|c|c|c|c|c|c|c|c|c|c|c|c|}
\hline \multirow{2}{*}{ Criteria } & \multirow{2}{*}{ Etching } & \multicolumn{4}{|c|}{ Baseline } & \multicolumn{4}{|c|}{3 months } & \multicolumn{4}{|c|}{6 months } \\
\hline & & A & B & $\mathrm{C}$ & $\% A+B$ & A & B & $\mathrm{C}$ & $\% A+B$ & A & B & $\mathrm{C}$ & $\% A+B$ \\
\hline \multirow{2}{*}{ Retention } & Yes & 76 & 0 & 0 & $100 \%$ & 72 & 0 & 4 & $94.7 \%$ & 64 & 0 & 8 & $84.2 \%$ \\
\hline & No & 74 & 0 & 0 & $100 \%$ & 68 & 0 & 6 & $94.1 \%$ & 57 & 0 & 11 & $77 \%$ \\
\hline \multirow{2}{*}{ Marginal integrity } & Yes & 76 & 0 & 0 & $100 \%$ & 68 & 0 & 4 & $94.4 \%$ & 60 & 3 & 1 & $93.7 \%$ \\
\hline & No & 74 & 0 & 0 & $100 \%$ & 63 & 0 & 5 & $92.6 \%$ & 50 & 5 & 2 & $87.7 \%$ \\
\hline \multirow{2}{*}{$\begin{array}{l}\text { Marginal discoloration } \\
\text { (absence) }\end{array}$} & Yes & 76 & 0 & 0 & $100 \%$ & 68 & 0 & 0 & $100 \%$ & 61 & 1 & 2 & $95.3 \%$ \\
\hline & No & 74 & 0 & 0 & $100 \%$ & 62 & 0 & 0 & $100 \%$ & 53 & 2 & 2 & $92.9 \%$ \\
\hline \multirow{2}{*}{ Tooth vitality } & Yes & 76 & 0 & 0 & $100 \%$ & 68 & 0 & 0 & $100 \%$ & 64 & 0 & 0 & $100 \%$ \\
\hline & No & 74 & 0 & 0 & $100 \%$ & 62 & 0 & 0 & $100 \%$ & 57 & 0 & 0 & $100 \%$ \\
\hline \multirow{2}{*}{ Postoperative sensitivity } & Yes & 76 & 0 & 0 & $100 \%$ & 68 & 0 & 0 & $0 \%$ & 64 & 0 & 0 & $0 \%$ \\
\hline & No & 74 & 0 & 0 & $100 \%$ & 62 & 0 & 0 & $0 \%$ & 57 & 0 & 0 & $0 \%$ \\
\hline \multirow{2}{*}{ Secondary caries } & Yes & 76 & 0 & 0 & $100 \%$ & 68 & 0 & 0 & $0 \%$ & 64 & 0 & 0 & $0 \%$ \\
\hline & No & 74 & 0 & 0 & $100 \%$ & 62 & 0 & 0 & $0 \%$ & 57 & 0 & 0 & $0 \%$ \\
\hline
\end{tabular}

TABLE 3: One-year evaluation of restorations.

\begin{tabular}{|c|c|c|c|c|c|c|c|c|c|}
\hline \multirow{2}{*}{ Criteria } & \multirow{2}{*}{ Etching } & \multicolumn{4}{|c|}{ Baseline } & \multicolumn{4}{|c|}{12 months } \\
\hline & & A & $\mathrm{B}$ & $\mathrm{C}$ & $\% \mathrm{~A}+\mathrm{B}$ & $\mathrm{A}$ & $\mathrm{B}$ & $\mathrm{C}$ & $\% \mathrm{~A}+\mathrm{B}$ \\
\hline \multirow{2}{*}{ Retention } & Yes & 76 & 0 & 0 & $100 \%$ & 39 & 0 & 4 & $90.6 \%$ \\
\hline & No & 74 & 0 & 0 & $100 \%$ & 30 & 0 & 9 & $76.9 \%$ \\
\hline \multirow{2}{*}{ Marginal integrity } & Yes & 76 & 0 & 0 & $100 \%$ & 31 & 6 & 2 & $94.8 \%$ \\
\hline & No & 74 & 0 & 0 & $100 \%$ & 17 & 10 & 3 & $90 \%$ \\
\hline \multirow{2}{*}{ Marginal discoloration (absence) } & Yes & 76 & 0 & 0 & $100 \%$ & 29 & 7 & 3 & $92.3 \%$ \\
\hline & No & 74 & 0 & 0 & $100 \%$ & 19 & 5 & 6 & $80 \%$ \\
\hline \multirow{2}{*}{ Tooth vitality } & Yes & 76 & 0 & 0 & $100 \%$ & 39 & 0 & 0 & $100 \%$ \\
\hline & No & 74 & 0 & 0 & $100 \%$ & 30 & 0 & 0 & $100 \%$ \\
\hline \multirow{2}{*}{ Postoperative sensitivity } & Yes & 76 & 0 & 0 & $100 \%$ & 39 & 0 & 0 & $100 \%$ \\
\hline & No & 74 & 0 & 0 & $100 \%$ & 30 & 0 & 0 & $100 \%$ \\
\hline \multirow{2}{*}{ Secondary caries } & Yes & 76 & 0 & 0 & $100 \%$ & 39 & 0 & 0 & $100 \%$ \\
\hline & No & 74 & 0 & 0 & $100 \%$ & 30 & 0 & 0 & $100 \%$ \\
\hline
\end{tabular}


TABLE 4: Statistical results (Mc Nemar' test).

\begin{tabular}{|c|c|c|c|c|c|c|}
\hline & Retention & Marginal integrity & Marginal discoloration & Tooth vitality & Postoperative sensitivity & Secondary caries \\
\hline Chi-square & 5.333 & & 5.333 & & & \\
\hline$P$ value & 0.021 & 0.000 & 0.021 & 0.093 & 0.093 & 0.093 \\
\hline
\end{tabular}

composite in the restoration of 150 NCCLs at baseline and after 3, 6, and 12 months.

Self-etch adhesive systems and composite resins permit restorations with minimally invasive preparation and acceptable aesthetic appearance. Most of the previous studies were conducted in vitro, and it is thus difficult to predict the clinical performance of these adhesives and resins. A long-term clinical study will provide the most accurate information regarding the durability of these adhesive restorations, but this type of study will need to span several years with regular recall visits and a high rate of recall for clinical validation. The clinical performance of two-step, self-etch adhesives has been evaluated previously [1]. NCCLs are commonly evaluated for a period of 3 to 5 years $[1,10]$. According to Blunck et al., a period of 6 months to 1 year can be sufficient to accurately predict the clinical behavior of an adhesive [15].

The current study evaluated the clinical performance of the Optibond All-in-One one-step, self-etch adhesive system. Of the USPHS criteria, four parameters were utilized to determine the overall clinical success, in accordance with Peumans et al. [9]: retention, marginal integrity, marginal discoloration, and tooth vitality.

Our results at 3 months following restoration showed retention rates of $94.7 \%$ and $94.1 \%$ with and without prior acid etching of the enamel margins, respectively. The 6month retention rate was $84.2 \%$ for restorations with etched enamel margins and $77 \%$ for those without acid etching. After one year, the recall rate was $58.06 \%$. As the missing patients may have lost their restorations, they did not attend the recall appointment. So, this fact may explain low retention rate obtained at 12 -month recall. The retention rates were $90.6 \%$ and $76.9 \%$ with and without acid etching, respectively. This improved performance of restorations with prior acid etching confirmed results reported by Burrow and Tyas [12]. In a one-year clinical trial of the All-in-One adhesive G-Bond, Burrow and Tyas [12] showed that, out of 47 NCCLs etched with phosphoric acid, all restorations were retained (100\% retention). However, according to da Costa et al. [16], enamel beveling may not be clinically relevant for the retention of composite restorations in NCCLs after 12 months. Can Say et al. found similar results after one year [17]. In a one-year trial with the same G-Bond adhesive system, Kurokawa et al. [18] also found a retention rate of $100 \%$; however, that study had a small sample size $(n=14)$.

Despite the limited clinical trial data available for All-inOne adhesive systems, the available studies provide promising information with regard to retention. Kubo et al. [14] used one-step self-etch adhesive systems (Clearfil S3 Bond and G-Bond) on 108 NCCLs with beveled enamel margins and ground dentin and found a retention rate of $98.1 \%$ after two years. The present study, which did not utilize any enamel or dentin preparations, showed a lower retention rate of $76.9 \%$.
Oral hygiene habits may have influenced the durability of the restorations. The majority of the Senegalese population uses sticks for teeth cleaning, which may have affected the retention of our restorations.

Marginal integrity defects (Code C) were observed at 3 months following restoration in 9 teeth (4 with and 5 without acid etching). The preservation of marginal integrity was $94.4 \%$ with acid etching and $92.6 \%$ without acid etching. Six months after restoration, the rates of marginal integrity preservation were $93.7 \%$ and $87.7 \%$ with and without acid etching, respectively. At the one-year recall, $94.8 \%$ of restorations with prior acid etching showed perfect marginal integrity. Kubo et al. [14] found $100 \%$ marginal integrity after 2 years. These findings indicate that marginal integrity in the range of $87.7 \%$ to $100 \%$ seems to be acceptable with or without enamel preparation.

No marginal discoloration was recorded at 3 months, whereas at the 6-month recall, marginal discoloration was absent in $92.9 \%$ of restorations without acid etching and in $95.3 \%$ of restorations with etched enamel margins. The results at one year revealed the absence of discoloration for $92.3 \%$ of cases with and $80 \%$ without acid etching. The one-year results from other clinical trials using the newly developed onestep self-etch systems showed almost no marginal staining $[12,16]$. After 2 years, Kubo et al. [14] reported slight marginal staining in 11 restorations using both Clearfil S3 Bond and GBond, which represented about $20 \%$ of the restorations, while Moretto et al. [19] reported a $1.2 \%$ rate of severe marginal defects after 3 years.

A better recall rate (100\%) was obtained by Tuncer et al. [20] at 6 and 12 months when using two different microhybrid composites and a two-step etch-and-rinse system in cervical restorations. A satisfactory clinical performance after 12 months was reported after 12 months.

In the present study, we did not observe any loss of tooth vitality. Peumans et al. [1] also described $100 \%$ pulp vitality in a study with the two-step self-etch adhesive Clearfil SE.

\section{Conclusion}

Based on modified USPHS criteria, restorations of NCCLs with the one-step, self-etch adhesive Optibond All-in-One in combination with the composite resin Herculite XRV Ultra with prior acid etching of the enamel margins demonstrated clinically acceptable results after one year. Retention was the most critical parameter in the protocol used in this study.

\section{Conflict of Interests}

The authors declare that there is no conflict of interests regarding the publication of this paper. 


\section{Acknowledgment}

The authors would like to thank the manufacturers for supplying materials for this study.

\section{References}

[1] M. Peumans, J. de Munck, K. Van Landuyt, P. Lambrechts, and B. Van Meerbeek, "Five-year clinical effectiveness of a two-step self-etching adhesive," Journal of Adhesive Dentistry, vol. 9, no. 1, pp. 7-10, 2007.

[2] B. Chee, L. J. Rickman, and J. D. Satterthwaite, "Adhesives for the restoration of non-carious cervical lesions: a systematic review," Journal of Dentistry, vol. 40, no. 6, pp. 443-452, 2012.

[3] J. de Munck, B. van Meerbeek, I. Satoshi et al., "Microtensile bond strengths of one- and two-step self-etch adhesives to burcut enamel and dentin," American Journal of Dentistry, vol. 16, no. 6, pp. 414-420, 2003.

[4] B. van Meerbeek, J. de Munck, D. Mattar, K. van Landuyt, and P. Lambrechts, "Microtensile bond strengths of an etch and rinse and self-etch adhesive to enamel and dentin as a function of surface treatment," Operative Dentistry, vol. 28, no. 5, pp. 647660, 2003.

[5] J. de Munck, M. Vargas, J. Iracki et al., "One-day bonding effectiveness of new self-etch adhesives to bur-cut enamel and dentin," Operative Dentistry, vol. 30, no. 1, pp. 39-49, 2005.

[6] M. Sarr, A. W. Kane, J. Vreven et al., "Microtensile bond strength and interfacial characterization of 11 contemporary adhesives bonded to bur-cut dentin," Operative Dentistry, vol. 35, no. 1 , pp. 94-104, 2010.

[7] B. Faye, A. W. Kane, M. Sarr, C. Lo, A. V. Ritter, and J. O. Grippo, "Noncarious cervical lesions among a non-toothbrushing population with Hansen's disease (leprosy): initial findings," Quintessence International, vol. 37, no. 8, pp. 613-619, 2006.

[8] J. O. Grippo, M. Simring, and T. A. Coleman, "Abfraction, abrasion, biocorrosion, and the enigma of noncarious cervical lesions: a 20-year perspective," Journal of Esthetic and Restorative Dentistry, vol. 24, no. 1, pp. 10-23, 2012.

[9] M. Peumans, P. Kanumilli, J. de Munck, K. van Landuyt, P. Lambrechts, and B. van Meerbeek, "Clinical effectiveness of contemporary adhesives: a systematic review of current clinical trials," Dental Materials, vol. 21, no. 9, pp. 864-881, 2005.

[10] S. Kubo, K. Kawasaki, H. Yokota, and Y. Hayashi, "Five-year clinical evaluation of two adhesive systems in non-carious cervical lesions," Journal of Dentistry, vol. 34, no. 2, pp. 97-105, 2006.

[11] J. W. V. van Dijken, "A randomized controlled 5-year prospective study of two hema-free adhesives, a 1-step self etching and a 3-step etch-and-rinse, in non-carious cervical lesions," Dental Materials, vol. 29, no. 11, pp. e271-e280, 2013.

[12] M. F. Burrow and M. J. Tyas, "Clinical trial of G-Bond all-inone adhesive and gradia direct resin composite in non-carious cervical lesions-results at 1 year," Journal of Dentistry, vol. 35, no. 7, pp. 623-625, 2007.

[13] R. B. Ermis, K. L. van Landuyt, M. V. Cardoso, J. de Munck, B. van Meerbeek, and M. Peumans, "Clinical effectiveness of a one-step self-etch adhesive in non-carious cervical lesions at 2 years," Clinical Oral Investigations, vol. 16, no. 3, pp. 889-897, 2012.

[14] S. Kubo, H. Yokota, H. Yokota, and Y. Hayashi, "Two-year clinical evaluation of one-step self-etch systems in non-carious cervical lesions," Journal of Dentistry, vol. 37, no. 2, pp. 149-155, 2009.

[15] U. Blunck, K. Knitter, and K.-R. Jahn, "Six-month clinical evaluation of XP BOND in noncarious cervical lesions," Journal of Adhesive Dentistry, vol. 9, no. 2, pp. 265-268, 2007.

[16] T. R. F. da Costa, A. D. Loguercio, and A. Reis, "Effect of enamel bevel on the clinical performance of resin composite restorations placed in non-carious cervical lesions," Journal of Esthetic and Restorative Dentistry, vol. 25, no. 5, pp. 346-356, 2013.

[17] E. Can Say, E. Özel, H. Yurdagüven, and M. Soyman, “Threeyear clinical evaluation of a two-step self-etch adhesive with or without selective enamel etching in non-carious cervical sclerotic lesions," Clinical Oral Investigations, vol. 18, no. 5, pp. 1427-1433, 2014.

[18] H. Kurokawa, M. Miyazaki, T. Takamizawa, A. Rikuta, K. Tsubota, and S. Uekusa, "One-year clinical evaluation of five singlestep self-etch adhesive systems in non-carious cervical lesions," Dental Materials Journal, vol. 26, no. 1, pp. 14-20, 2007.

[19] S. G. Moretto, E. M. A. Russo, R. C. R. Carvalho et al., "3-year clinical effectiveness of one-step adhesives in non-carious cervical lesions," Journal of Dentistry, vol. 41, no. 8, pp. 675-682, 2013.

[20] D. Tuncer, Ç. Çelik, K. Yamanel, and N. Arhun, "1 year clinical evaluation of microhybrid composites used in the restoration of non-carious cervical lesions," Oral Health and Dental Management, vol. 13, no. 2, pp. 366-371, 2014. 


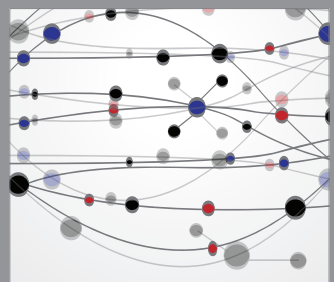

The Scientific World Journal
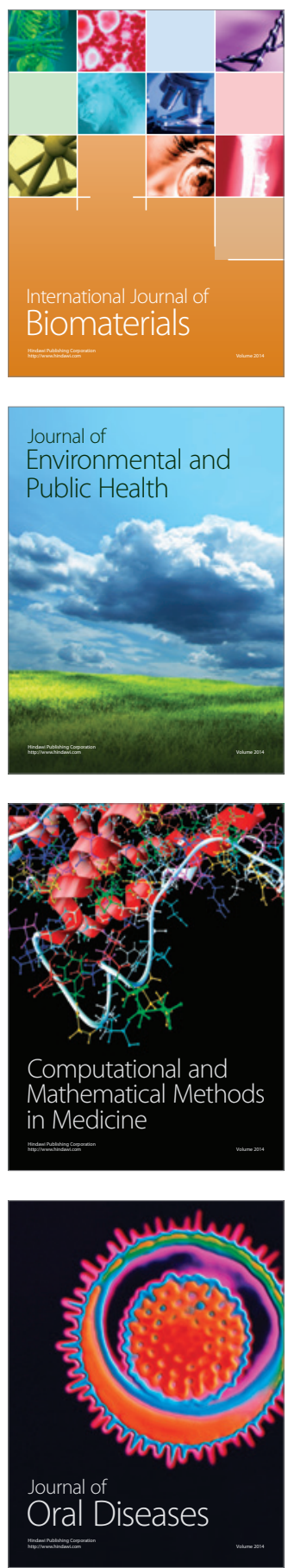
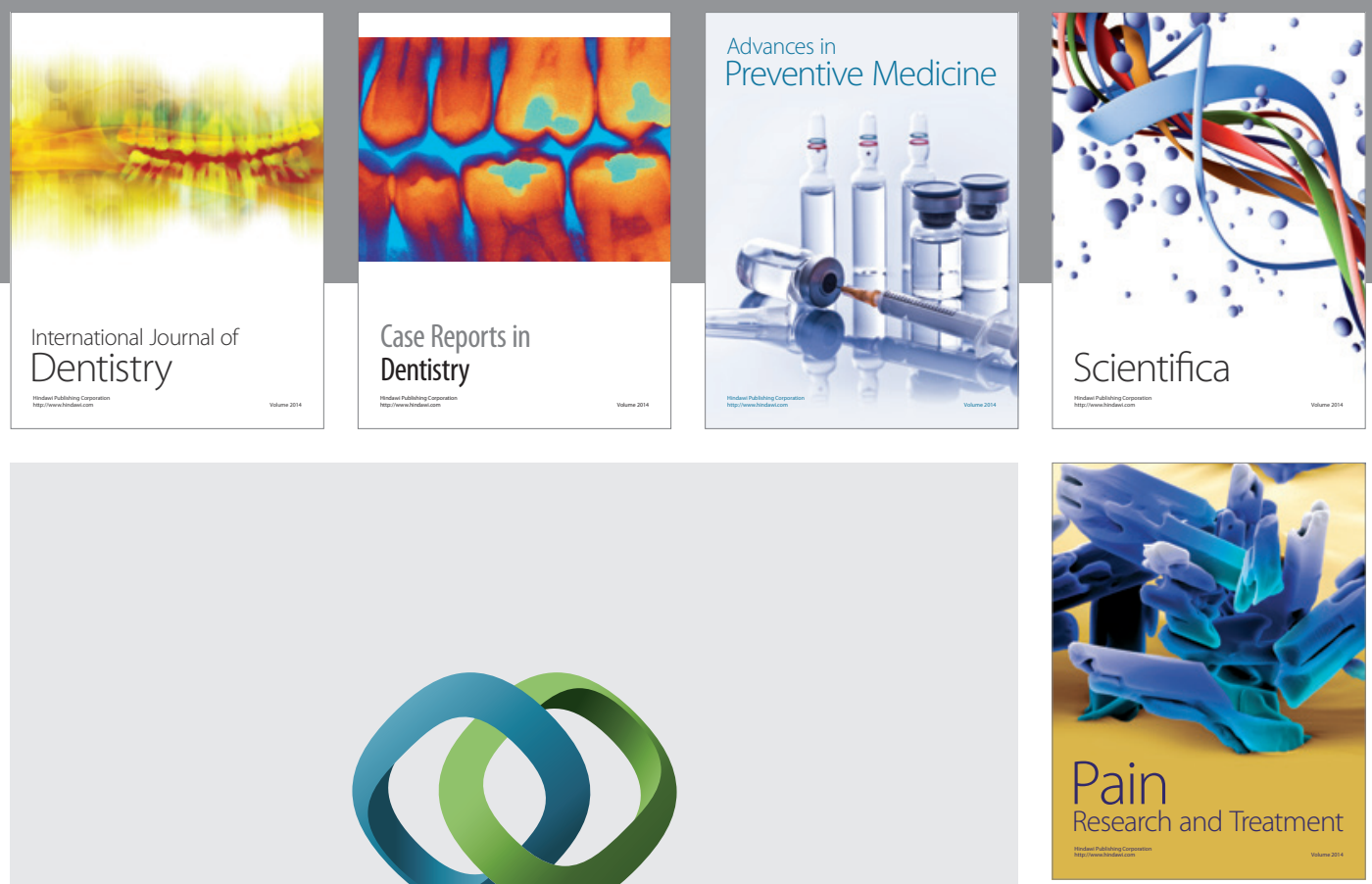

\section{Hindawi}

Submit your manuscripts at

http://www.hindawi.com
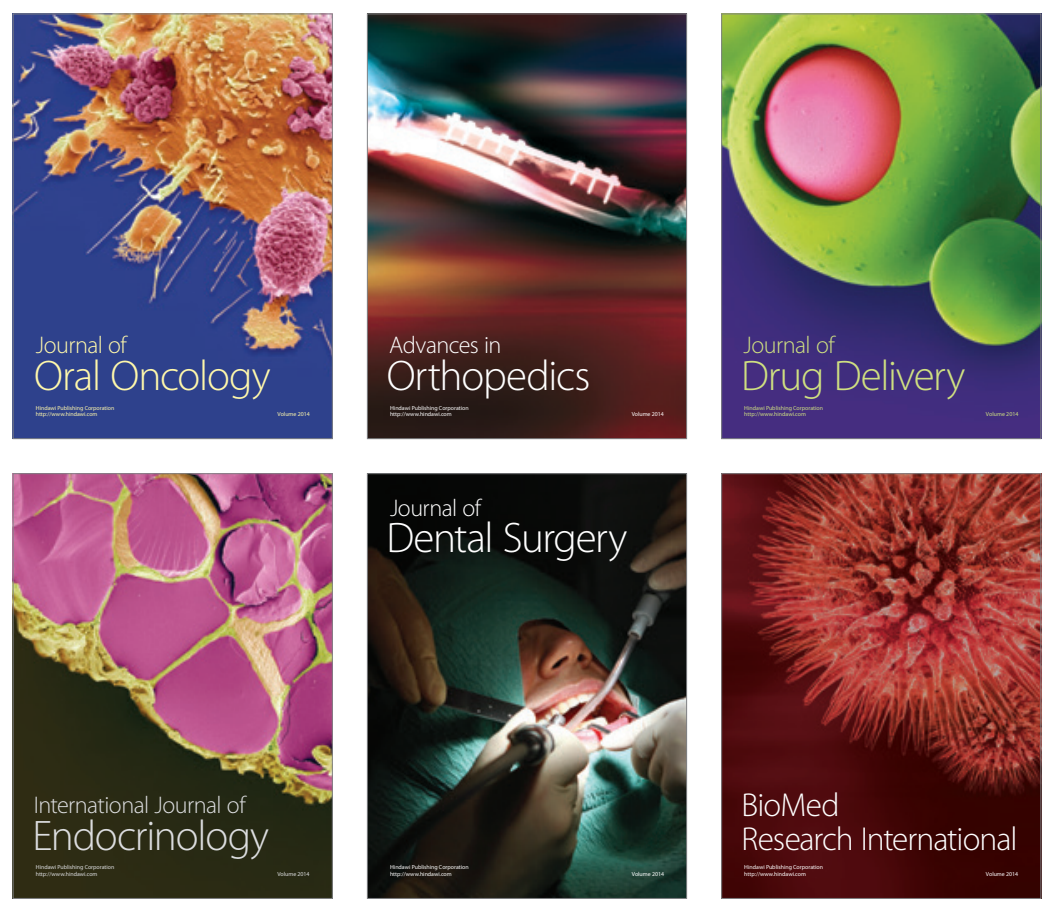

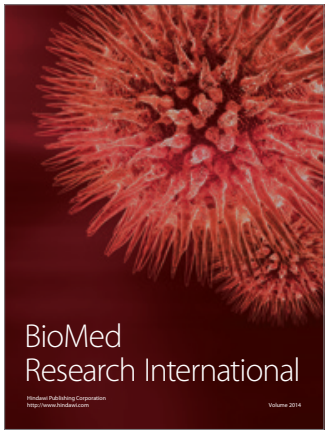

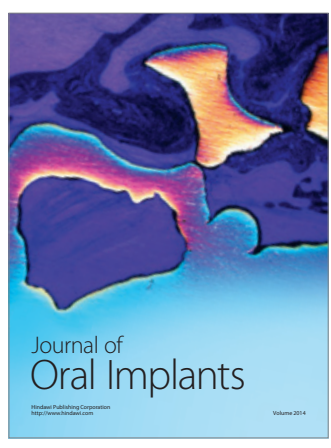
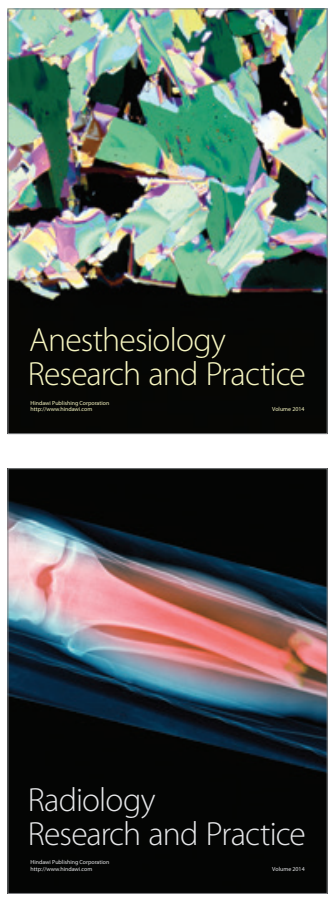